\title{
Circulating miR-451a levels as a potential biomarker to predict the prognosis of patients with multiple myeloma
}

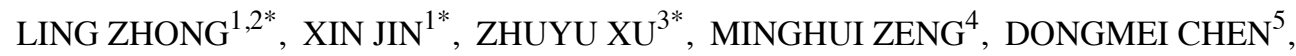 \\ YUAN HE $^{1}$, JIANBO ZHANG ${ }^{1}$, TAO JIANG ${ }^{6}$ and JIAO CHEN ${ }^{6}$ \\ ${ }^{1}$ Department of Clinical and Experimental Medicine, Sichuan Academy of Medical Sciences and \\ Sichuan Provincial People's Hospital, School of Medicine, University of Electronic Science and Technology of China, \\ Chengdu, Sichuan 610072; ${ }^{2}$ Department of Natural Products Research Center, Chengdu Institute of Biology, \\ Chinese Academy of Sciences, Chengdu, Sichuan 610041; ${ }^{3}$ Department of Pharmacy, Sichuan Academy of \\ Medical Science and Sichuan Provincial People's Hospital, Personalized Drug Therapy Key Laboratory of Sichuan Province, \\ School of Medicine, University of Electronic Science and Technology of China, Chengdu, Sichuan 610072; \\ ${ }^{4}$ Department of Pharmacy, Qionglai Municipal Medical Center Hospital of Sichuan Province, Chengdu, Sichuan 611530; \\ ${ }^{5}$ Department of Clinical and Experimental Medicine, Southwest Medical University Clinical Medical School, Luzhou, \\ Sichuan 646000; ${ }^{6}$ Department of Hematology, Sichuan Academy of Medical Sciences and \\ Sichuan Provincial People's Hospital, School of Medicine, University of Electronic Science \\ and Technology of China, Chengdu, Sichuan 610072, P.R. China
}

Received December 24, 2019; Accepted July 22, 2020

DOI: $10.3892 / \mathrm{ol} .2020 .12126$

\begin{abstract}
The natural course of multiple myeloma (MM) varies greatly between patients. The Revised MM International Staging System (R-ISS) identifies high-risk patients, but it is unsuitable for assessing minimal residual disease (MRD). Furthermore, the focal location of myeloma cells and clonal evolution often produce false negative results in flow cytometry. Extracellular microRNA (miRNA/miR) expression levels are stable in bodily fluids, and are retrievable and measurable from fresh or archived serum or plasma samples. Therefore, the present study aimed to investigate the clinical utility of circulating miRNA levels in patients with MM, particularly miR-451a, which is commonly downregulated in MM, and whether it could predict the prognosis and relapse of patients with MM. In total, 66 patients with MM, stratified using the R-ISS criteria, were recruited, while 10 healthy subjects (transplantation donors) were enrolled as
\end{abstract}

Correspondence to: Professor Tao Jiang or Professor Jiao Chen, Department of Hematology, Sichuan Academy of Medical Sciences and Sichuan Provincial People's Hospital, School of Medicine, University of Electronic Science and Technology of China, 32 First Ring Road, Chengdu, Sichuan 610072, P.R. China

E-mail: suyeku@126.com

E-mail: chenjiaoxy@med.uestc.edu.cn

*Contributed equally

Key words: circulating, microRNA-451a, multiple myeloma, prognosis, relapse controls. Reverse transcription-quantitative PCR was used to evaluate miR-451a expression in bone marrow (BM) and in the circulation. IL-6 levels were measured using ELISA, while western blotting was conducted to analyze the protein expression levels of the IL-6 receptor (IL-6R). During follow-up, MRD was assessed via multiparameter flow cytometry (MFC). miR-451a was identified to target IL-6R using a dual-luciferase reporter assay. Circulating miR-451a levels were low in patients with MM, and was found to be 0.39 times that of the control group ( $\mathrm{U}=4.00 ; \mathrm{P}<0.001)$. Among the 66 patients with $\mathrm{MM}$, the median level of miR-451a was 0.73 and 0.41 times that of the control group in R-ISS stage I MM (15 patients) and R-ISS stage II stage (17 patients), respectively; patients with R-ISS stage III MM (34 patients) had the lowest level, at 0.24 times the value of the control group. Circulating miR-451a levels had a strong positive correlation with miR-451a levels in BM, but negatively correlated with IL-6 and IL-6R levels. After two courses of consolidation chemotherapy, 19 patients achieved complete remission, 10 of whom presented steady circulating miR-451a levels during follow-up; the other nine patients had an abrupt decrease in circulating miR-451a levels. The turning points in the trend appeared 4-8 weeks before positive results were obtained via MFC, and 4-16 weeks before clinical relapse. Moreover, miR-451a overexpression notably downregulated the expression of the IL-6R mRNA and protein. Collectively, circulating miR-451a levels potentially represent a novel biomarker to monitor MRD and predict relapse.

\section{Introduction}

Multiple myeloma (MM) is an incurable cancer of plasma cells caused by the abnormal accumulation of monoclonal plasma 
cells in the bone marrow (BM) (1), which accounts for $\sim 10 \%$ of all hematological malignancies (2). The natural course of MM varies greatly. For example, some patients live for only a few months, while other patients survive for decades (3). The identification patients who are at high risk may help optimize the choice of personalized treatment and improve clinical outcomes (3). Currently, the Revised MM International Staging System (R-ISS) is widely used in clinics for stratification and can successfully classifies patients (3). The R-ISS staging system consists of determining $\beta_{2}$-microglobulin $\left(\beta_{2}-\mathrm{M}\right)$, albumin (ALB), lactate dehydrogenase (LDH) and cytogenetic heterogeneity (4). In addition to stratification, dynamically monitoring of the effect of therapy, termed 'minimal residual disease' (MRD), is important for predicting relapse and the prognosis in order to intervene in a timely manner and prolong survival (5). However, the R-ISS is not suitable for assessing MRD. For example, the cytogenetic heterogeneity does not dynamically reflect the tumor load at different stages of treatment. Moreover, diverse genetic abnormalities exist in different patients, and numerous factors influence $\beta_{2}-\mathrm{M}$, ALB and LDH levels (5).

Currently, multiparameter flow cytometry (MFC) of the BM is routinely used for monitoring MRD (6). However, MM has a unique characteristic, namely, myeloma cells are not evenly distributed in BM (foci), and this characteristic often causes false negative results for MRD (7). Therefore, identifying a novel marker that is universal for the majority of patients, that reflects the dynamic changes in tumor load and is able to be conveniently assessed is urgently needed. For these purposes, the present study aimed to develop a non-invasive approach.

IL-6 is a pivotal growth factor that regulates the proliferation of MM via an autocrine mechanism from MM cells or a paracrine mechanism from stromal cells (8). IL-6 exerts its biological effects by binding to its receptor (IL-6R), and IL-6R leads to the activation of the JAK/STAT signaling pathway (9). The activation of JAK family tyrosine kinases recruits the STAT3 protein, which dimerizes and translocates to the nucleus to transduce signals and initiate the expression of genes involved in the proliferation and apoptosis of myeloma cells (10).

MicroRNAs (miRNAs/miRs) can identify the 3 ' untranslated region (3'UTR) of their specific target mRNAs and subsequently inhibit the expression of the target gene at the post-transcriptional level (11). miR-451a was discovered in 2005 and is located on chromosome 17qll.2 (11). Furthermore, miR-451a, which functions as a tumor suppressor, has been reported to be associated with several types of cancer, including lung cancer (12), hepatocellular carcinoma (13), gliomas (14) and osteosarcoma (15), wherein it inhibits the proliferation, migration and invasion of tumors (16). miR-451a has also been shown to target IL-6R in several solid tumor types, and inhibits tumor proliferation, migration and angiogenesis via the IL-6R signaling pathway (17). However, whether miR-451a inhibits tumor growth in MM remains unknown, and whether there a targeted relationship between miR-451a and IL-6R in $\mathrm{MM}$ is yet to be elucidated. Moreover, further investigations are required to determine whether circulating miR-451a levels reflect the tumor load in real time. The current study aimed to evaluate these questions, and the present findings may provide a potential prognostic strategy for MM.

\section{Materials and methods}

Ethics statement. This study was performed with the approval of the Institutional Review Board of Sichuan Provincial People's Hospital of China. Informed written consent was collected from each eligible patient, and the whole study was performed in accordance with the Declaration of Helsinki.

Study subjects. Between January 2017 and December 2018, 66 patients with MM (age range, 38-88 years; 37 men; 29 women) and ten healthy controls (age range, 31-83 years; six men; four women), who were treated at the Sichuan Academy of Medical Sciences and Sichuan Provincial People's Hospital were enrolled. The diagnostic criteria for active MM were based on the International Myeloma Working Group guidelines (18). The criteria for classification were according to the subtype of abnormal proliferative immunoglobulin. The criteria for staging were assessed using the R-ISS (3). The healthy control group consisted of transplant donors undergoing BM transplantation, who were recruited to provide $\mathrm{BM}(\sim 1 \mathrm{ml})$ and peripheral blood samples $(\sim 5 \mathrm{ml})$.

Treatment. All patients were treated with bortezomib $\left(1.3 \mathrm{mg} / \mathrm{m}^{2}\right.$ subcutaneously on days $1,4,8$ and 11 of every 21-day cycle or $1.3 \mathrm{mg} / \mathrm{m}^{2}$ subcutaneously on days $1,8,15$ and 22 of every 35-day cycle; three manufacturers permitted by medical insurance: QILU Pharmaceutical; Chia Tai Tianqing Pharmaceutical; Hansoh Pharmaceutical) in combination with dexamethasone (Tianjin Jinyao Pharmaceutical Co., Ltd.; 10 or $20 \mathrm{mg}$ on the day of, and the day after the administration of each dose of bortezomib). In high-risk patients, either cyclophosphamide $\left(300 \mathrm{mg} / \mathrm{m}^{2}\right.$ on days 1 and 8; Baxter Oncology $\mathrm{GmbH}$ ) or lenalidomide (10 or $25 \mathrm{mg}$ based on renal function measured from days 1-21; Beijing Shuanglu Pharmaceutical Co., Ltd.) was added to the combination. A total of 8-12 cycles were planned as induction therapy for all patients, and after induction therapy, patients entered maintenance therapy with either bortezomib $\left(1.3 \mathrm{mg} / \mathrm{m}^{2}\right.$ once every 2 weeks for 2 years $)$ or lenalidomide (25 mg once a day on days 1-21 and every 28 days until relapse).

Sampling. Samples were initially collected from patients at the first diagnosis and at the required follow-up time points (once every 3 months); $5 \mathrm{ml}$ blood was collected in serum collection tubes. Whole blood was allowed to stand for $\sim 1 \mathrm{~h}$ at room temperature, before centrifugation at $1,200 \mathrm{x}$ g for $10 \mathrm{~min}$ at $4^{\circ} \mathrm{C}$, followed by the separation of serum. Successive centrifugation was performed for $10 \mathrm{~min}$ at $10,000 \mathrm{xg}$ at $4^{\circ} \mathrm{C}$ to remove the cellular debris. The resulting serum was aliquoted into Eppendorf tubes and stored at $-80^{\circ} \mathrm{C}$ to determine IL-6 and circulating miR-451a levels.

BM samples collected from both patients and controls were anticoagulated with EDTA- $\mathrm{K}_{2}$ (BD Biosciences) at room temperature. Some BM samples were diluted 1:1 with RPMI-1640 basic medium (Gibco; Thermo Fisher Scientific, Inc.). Mononuclear cells were isolated with Ficoll cell isolation medium at room temperature (density $1.077 \mathrm{~g} / \mathrm{ml}$; Stemcell Technologies, Inc.). Then, plasma cells were isolated using CD138 magnetic beads at room temperature (Miltenyi Biotec, Inc.) for RNA extraction to analyze miR-451a expression 
and proteins were extracted to measure IL-6R levels. The remaining $\mathrm{BM}$ was used for MFC.

\section{Analysis}

Reverse transcription-quantitative PCR (RT-qPCR). To concentrate the circulating miRNA, the extraction protocol was conducted by adding $10 \%$ PEG 8,000 solution (Beijing Solarbio Science \& Technology Co., Ltd.). Total RNA was extracted from $500 \mu \mathrm{l}$ serum using a mirVana miRNA Isolation kit (cat. no. AM1561; Thermo Fisher Scientific, Inc.), according to the manufacturer's instructions. Then, miR-451a was reverse transcribed with a Bulge-LoopTM miRNA RT-qPCR Starter kit (Guangzhou RiboBio Co., Ltd.). The reaction conditions were: $42^{\circ} \mathrm{C}$ for $60 \mathrm{~min}$ and $70^{\circ} \mathrm{C}$ for $10 \mathrm{~min}$. Relative quantification was performed via qPCR using the SYBR Green PCR kit (Guangzhou RiboBio Co.,Ltd.). External references (cel-miR-39; Guangzhou RiboBio Co., Ltd.) were used to compare miRNA levels among different groups. The fold change was calculated based on the normalized mean differences $\left(2^{-\Delta \Delta \mathrm{Cq}}\right)$ (19). The PCR cycling conditions were as follows: Initial denaturation at $95^{\circ} \mathrm{C}$ for $10 \mathrm{~min}$, followed by 40 cycles at $95^{\circ} \mathrm{C}$ for $2 \mathrm{sec}, 60^{\circ} \mathrm{C}$ for $20 \mathrm{sec}$ and $70^{\circ} \mathrm{C}$ for $10 \mathrm{sec}$. The following primers for miR-451a were used: Sense, $5^{\prime}-\mathrm{ACC}$ GTTACCATTACT-3' and antisense, 5'-CTCACACGACTC ACGA-3'. All reactions, including no-template controls, were performed in triplicate. Amplification and data analysis were performed using an ABI Prism 7,000 thermocycler (Applied Biosystems; Thermo Fisher Scientific, Inc.).

Primary plasma cells were purified from BM aspirates obtained from patients with MM and healthy volunteers using CD138 magnetic beads (Miltenyi Biotec, Inc.). Total RNA of cells was extracted using Tripure isolation reagent (Roche Diagnostics, Inc.) and reverse transcribed into cDNAs with a first stand cDNA synthesis kit using random primers (Roche Diagnostics). The duration of RT was as following: $25^{\circ} \mathrm{C}$ for $10 \mathrm{~min}, 55^{\circ} \mathrm{C}$ for $30 \mathrm{~min}, 85^{\circ} \mathrm{C}$ for $5 \mathrm{~min}, 10^{\circ} \mathrm{C}$ for $15 \mathrm{~min}$ and end at $4^{\circ} \mathrm{C}$. qPCR was performed to analyze IL-6R expression using SYBR Premix Ex Taq (Takara Bio, Inc.). The qPCR consisted of an initial denaturation step at $95^{\circ} \mathrm{C}$ for $5 \mathrm{~min}$, followed by 39 cycles at $95^{\circ} \mathrm{C}$ for $10 \mathrm{sec}, 60^{\circ} \mathrm{C}$ for $30 \mathrm{sec}$, and a final elongation step at $95^{\circ} \mathrm{C}$ for $15 \mathrm{sec}, 60^{\circ} \mathrm{C}$ for $1 \mathrm{~min}$ and $95^{\circ} \mathrm{C}$ for $15 \mathrm{sec}$. The following primers for IL-6R were used: Sense, 5'-CCTCTGCATTGCCATTGTTC-3' and antisense, 5'-GAGATGAGAGGAACAAGCAC-3'.

ELISA. The serum level of IL-6 in serum was measured using a human IL-6 ELISA kit (Abcam; cat. no. ab46027), according to the manufacturer's instructions. After color development was stopped, the absorbance was measured using a microtiter plate-computerized reader set to a wavelength at $450 \mathrm{~nm}$. The sensitivity for IL-6 was $2 \mathrm{pg} / \mathrm{ml}$.

Western blotting. Western blotting was used to analyze the levels of IL-6R in plasmocytes. Proteins were extracted from cells with a lysis buffer containing PMSF (Beijing Solarbio Science \& Technology Co., Ltd.) and concentrations were measured using a BCA protein assay kit (Tiangen Biotech Co., Ltd.). A total of 20-30 $\mu \mathrm{g}$ protein extracts were subjected to electrophoresis on $10 \%$ SDS-PAGE gels and transferred to nitrocellulose membranes. The membranes were blocked with
Table I. Patient characteristics.

\begin{tabular}{lc}
\hline Characteristics & Patients \\
\hline Sex & \\
Male & $37(56.1 \%)$ \\
Female & $29(43.9 \%)$ \\
Age, years & $63(38-88)$ \\
Isotype & \\
IgG type & $35(53.0 \%)$ \\
IgA type & $17(25.8 \%)$ \\
IgM type & 0 \\
IgE type & 0 \\
IgD type & $1(1.5 \%)$ \\
Light chain type & $13(19.7 \%)$ \\
R-ISS Stage & \\
I & $15(22.7 \%)$ \\
II & $17(25.8 \%)$ \\
III & $34(51.5 \%)$ \\
Cytogenetics & \\
Gain 1q21 & $12(13.6 \%)$ \\
17p deletion & $6(9.0 \%)$ \\
t (11;14) & $8(12.1 \%)$ \\
t (14;16) & $4(6.0 \%)$ \\
t (4;14) & $5(7.6 \%)$ \\
t (14;20) & $1(1.5 \%)$ \\
Trisomy & $27(40.9 \%)$ \\
Unknown & $3(4.5 \%)$ \\
LDH level exceeding the normal range & $11(16.7 \%)$ \\
& \\
& \\
&
\end{tabular}

Data are presented as the $\mathrm{n}(\%)$ or median (interquartile ranges). LDH normal range 120-250 U/1. LDH, lactate dehydrogenase; R-ISS, Revised Multiple Myeloma International Staging System.

$10 \%$ skimmed milk for $2 \mathrm{~h}$ at room temperature and then incubated with the following primary antibodies overnight at $4{ }^{\circ} \mathrm{C}$ : Rabbit anti-IL-6R (1:400; Abcam; cat. no. ab27321) and rabbit anti-GAPDH (1:1,000; Abcam; cat. no. ab8245). After incubation with the secondary antibody conjugated with horseradish peroxidase (1:2,000; Abcam; cat. no. ab6789) for $2 \mathrm{~h}$ at room temperature, the proteins were visualized with the Molecular Imager Gel Doc XR System (Bio-Rad Laboratories, Inc.) using Metal Enhanced DAB Substrate kit (Beijing Solarbio Science \& Technology Co., Ltd.).

Flow cytometry. In follow-up experiments, MRD was assessed using MFC. A fixative-free erythrocyte lysis buffer (Beckman Coulter, Inc.) was used for the phenotypic characterization of the majority of participants. Intraprep permeabilization reagent (cat. no. A07803; Beckman Coulter, Inc.) was used to analyze intracellular immunoglobulin levels at room temperature. The number and viability of cells obtained were determined using staining cells with trypan blue for $10 \mathrm{~min}$ at room temperature and counted under a microscope. If the viability of recovered cells was $\geq 90 \%, 20$ million cells were stained with two independent 6 -color panels (10 million each): One tube contained 
A
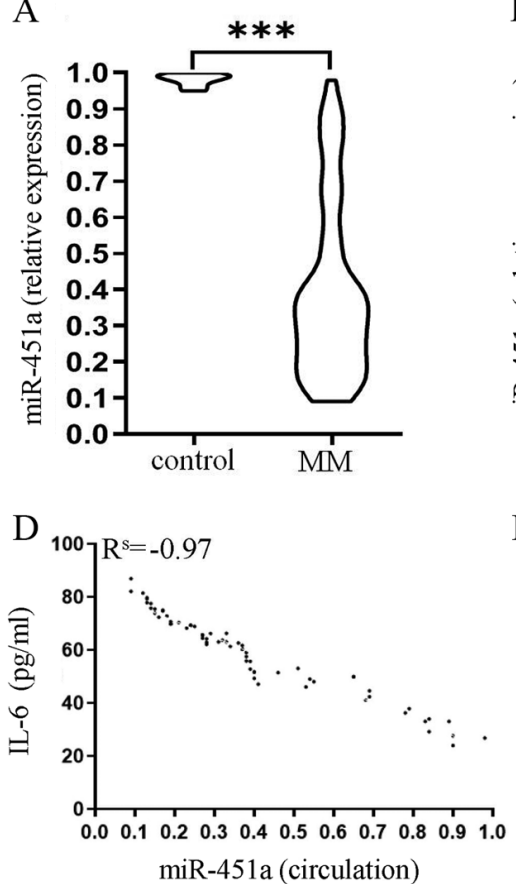

B

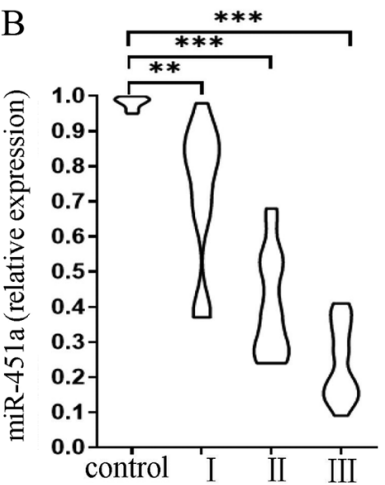

E

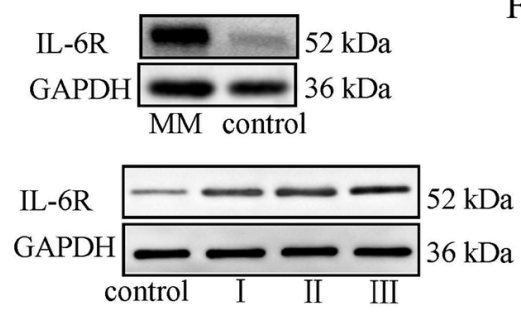

$\mathrm{C}$

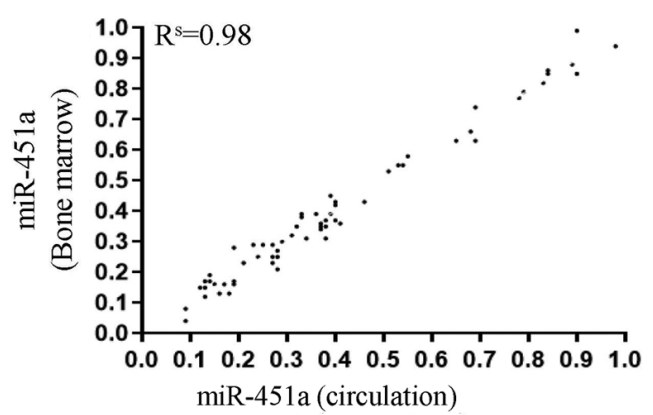

F

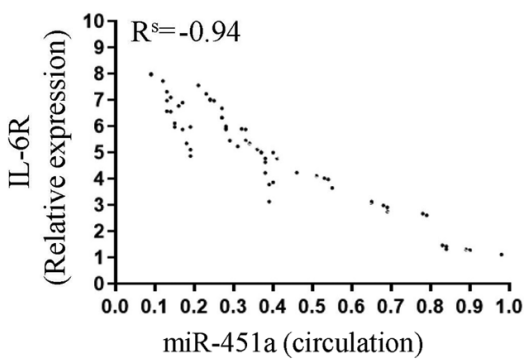

Figure 1. Expression of miR-451a in the BM and circulation of patients with MM. (A) Relative expression of miR-451a in healthy controls and patients with MM. (B) Expression of miR-451a among different Revised Multiple Myeloma International Staging System stages. (C) Correlation between miR-451a expression in circulation and in BM. (D) Correlation between circulating miR-451a and IL-6. (E) Western blotting results of the protein expression of IL-6R in plasma cells. (F) Correlation between circulating miR-451a and IL-6R. ${ }^{* *} \mathrm{P}<0.01,{ }^{* * *} \mathrm{P}<0.001$. miR, microRNA; MM, multiple myeloma; BM, bone marrow; IL-6R, IL-6 receptor.

CD19-FITC, CD20-PE, CD56-ECD, CD38-PECY5.5, CD138-APC and CD45-PECy7, and the other tube contained CD19-FITC, CD117-PE, CD56-ECD, CD38-PECY5.5, CD138-APC and CD45-PECy7. The intracytoplasmic panel consisted of the markers Сук-APC, Су $\lambda$-APC750, CD19-PE, CD38-FITC and CD138-APC. In total, 10 million cells suspended at a final volume of $200 \mu \mathrm{l} /$ tube were labeled in each tube. A minimum of 5 million events were recorded per tube in the mode of middle speed in a flow cytometer (Navios' Beckman Coulter, Inc.). The software used for analysis was Kaluza (version no. 2.1; Beckman Coulter, Inc.). Cell populations are considered abnormal if they have an atypical differentiation pattern, an increased or decreased expression level of normal antigens, an asynchronous maturational pattern or express aberrant antigens (20).

miR-451a transfection. For miR-451a mimic transfections, the miR-451a mimic (5'-AAACCGUUACCAUUACUGAGUU-3'; $50 \mathrm{nM}$; Guangzhou RiboBio Co., Ltd.) and the corresponding negative control (50 nM; Guangzhou RiboBio Co., Ltd.) were separately transfected into 293T cells (purchased from China Center for Type Culture Collection) were performed separately in the absence of any other treatments. The transfections were performed using Lipofectamine 3000 (Thermo Fisher Scientific, Inc.). Then, $48 \mathrm{~h}$ after transfection, the expression of miR-451a was measured using RT-qPCR to confirm successful transfections, and the subsequent effect was only determined in cells expressing miR-451a.

Luciferase reporter assay. The online software TargetScan (TargetScan Human 7.0; http://www.targetscan.org/vert_70/) was used to investigate the relationship between miR-451a and IL-6R. The predicted binding site for miR-451a to human IL-6R 3'UTR was 5'-UUGCCAAA-3' (17,21). The human IL-6R 3'UTR with a mutation in the miR-451a seed sequence (mutant; mut) and 3'UTR (wild-type; WT) were amplified and inserted between the restriction sites XhoI and NotI of the firefly and Renilla luciferase reporter vector pmiR-RB-REPORT (Guangzhou RiboBio Co., Ltd.), respectively. Cells were plated on 24 -well plates at $1 \times 10^{5}$ cells/well and transfected with luciferase reporter vectors (30 ng) using Lipofectamine 3,000 (Thermo Fisher Scientific, Inc.). At $24 \mathrm{~h}$ post-transfection, firefly and Renilla luciferase activities were measured using a Dual-Glo Luciferase assay system (Promega Corporation), according to the manufacturer's instructions. The Renilla luciferase signal was normalized to that of the firefly luciferase signal for each individual analysis.

Statistical analysis. Data are presented as the medians and interquartile ranges. The experiments were repeated three times. The Kolmogorov-Smirnov test was used to analyze whether the data were normally distributed. The Kruskal-Wallis rank sum test was used to analyze differences in the data among the four groups. Dunn's test was used as the post hoc test after Kruskal Wallis. Comparisons between two groups were performed using the Mann-Whitney U test. After the Mann-Whitney U test, a Bonferroni was conducted to correct the P-value. Correlations were determined by calculating Spearman's correlation coefficients. Statistical analyses were performed using SPSS version 19.0 (IBM Corp.). $\mathrm{P}<0.05$ was considered to indicate a statistically significant difference. 
A

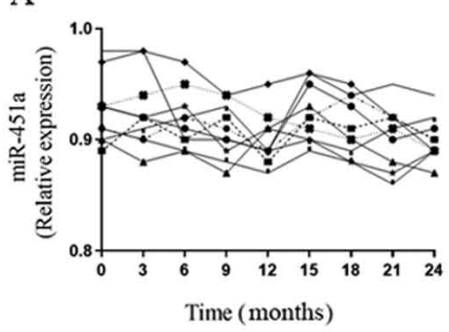

B

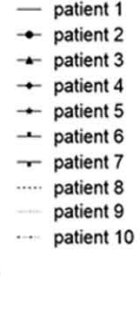

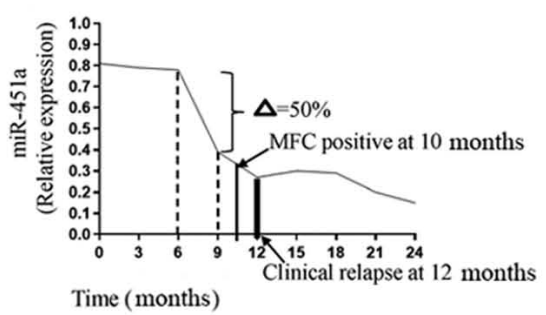

C

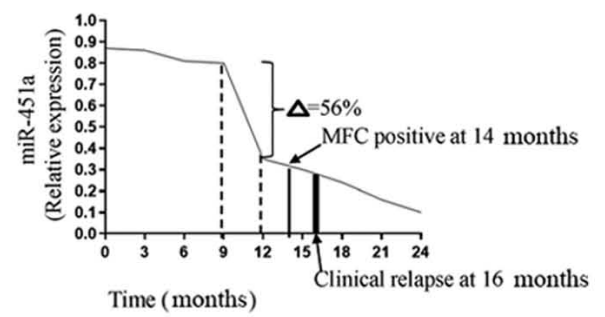

D

E
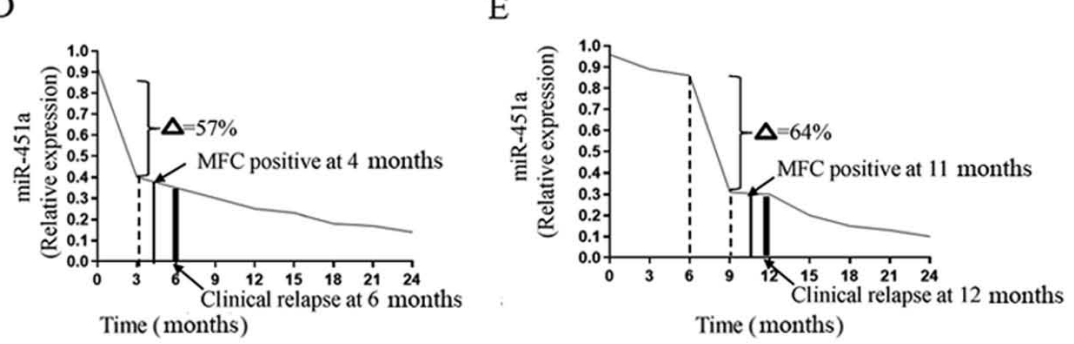

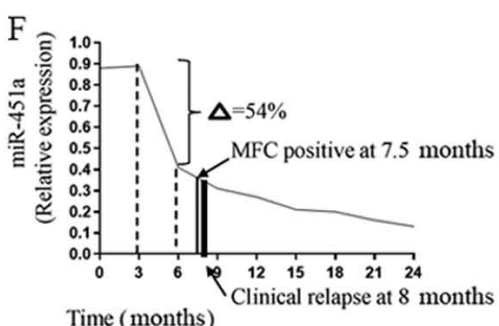

Time (months)
G

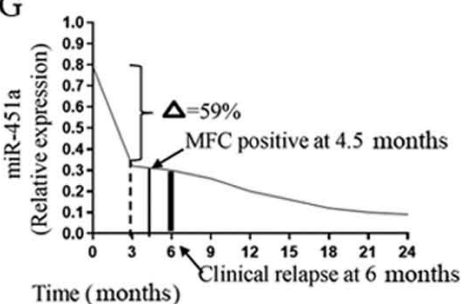

$\mathrm{H}$

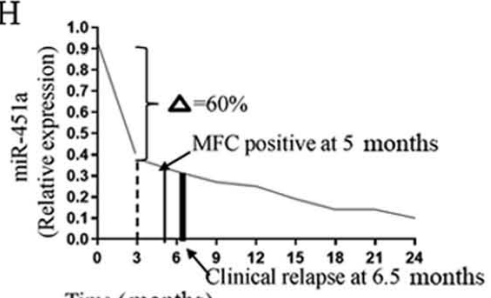

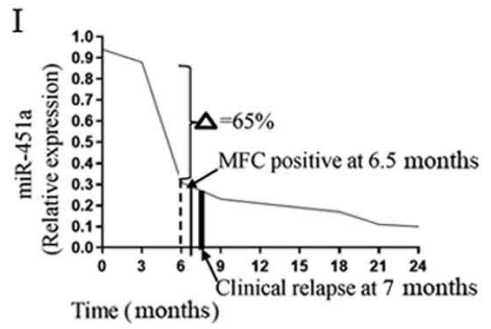

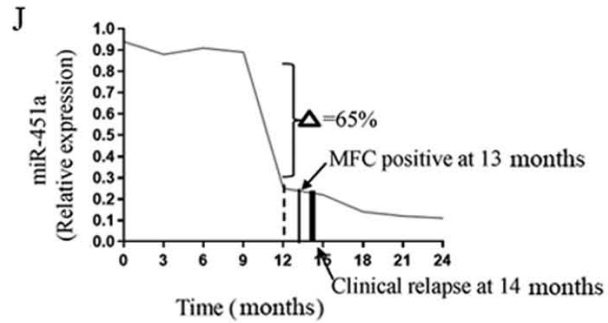

Figure 2. Follow-up of patients who experienced CR. (A) In total, 10 patients experienced long-term remission. (B-J) In total, nine patients relapsed during the follow-up period. (B) During the follow-up period, the expression of miR-451a at the 9th month after CR decreased by $50 \%$ compared with the value at the 6 th month $(\delta=50 \%)$. MRD was reported positive using MFC at the 10th month. The patient experienced a clinical relapse at the 12th month. (C) MFC positive at the 14th month, clinical relapse at the 16th month, $\delta=56 \%$. (D) $\delta=57 \%$, MFC positive at the 4 th month, and clinical relapse at the 6th month. (E) $\delta=64 \%$, MFC positive at the 11th month, and clinical relapse at the 12th month. (F) $\delta=54 \%$, MFC positive at the 7.5th month, and clinical relapse at the 8th month (G) $\delta=59 \%$, MFC positive at the 4.5th month, and clinical relapse at the 6th month. $(\mathrm{H}) \delta=60 \%$, MFC positive at the 5th month, and clinical relapse at the 6.5th month. (I) $\delta=65 \%$, MFC positive at the 6.5 th month, and clinical relapse at the 7 th month. (J) $\delta=65 \%$, MFC positive at the 13 th month, and clinical relapse at the14th month. CR, complete remission; MFC, multiparameter flow cytometry; miR, microRNA.

\section{Results}

Baseline characteristics and treatments. In the present study, the median age of the patients was 63.0 years (age range, $38-88$ years), and $56.1 \%$ of patients were male. The patients received immunomodulatory drugs or proteasome inhibitors as induction therapy without autologous stem cell transplantation. In total, 15 of these patients $(22.7 \%)$ were R-ISS stage I, 17 patients $(25.8 \%)$ were R-ISS stage II and 34 patients $(51.5 \%)$ were R-ISS stage III (Table I).

Expression of miR-451a in the BM and circulation of patients with MM. Low circulating miR-451a levels were detected in patients with $\mathrm{MM}$, and were only 0.39 times the value of the control group $(\mathrm{U}=4.00 ; \mathrm{P}<0.001$; Fig. 1A). Among the 66 patients with MM, the median expression of miR-451a was 0.73 times the value of the control group in R-ISS stage I MM; and that in R-ISS stage II MM was 0.41 times the value of the control group (Fig. 1B). Moreover, patients with R-ISS stage III MM presented the lowest level, at 0.24 times the value of the control group (Fig. 1B).

The expression of miR-451a was compared between cells in the BM and circulation to evaluate the consistency. The expression of miR-451a in the circulation exhibited a strong positive correlation with miR-451a expression in $\mathrm{BM}\left(\mathrm{R}^{\mathrm{s}}=0.98\right.$; $\mathrm{P}<0.001$; Fig. 1C). The concentration of IL-6 was strongly, 

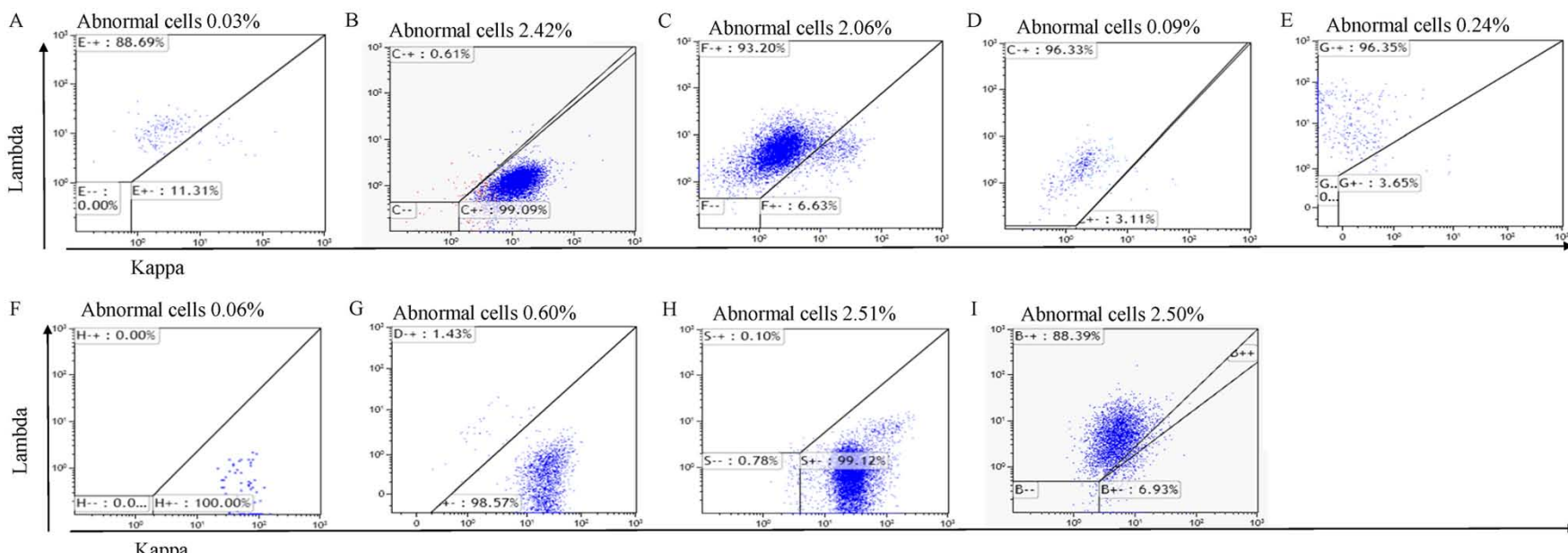

G Abnormal cells $0.60 \%$

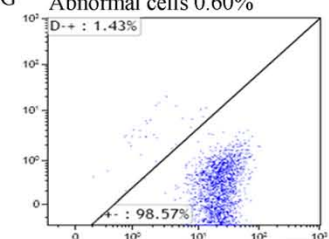

H Abnormal cells $2.51 \%$

I Abnormal cells $2.50 \%$
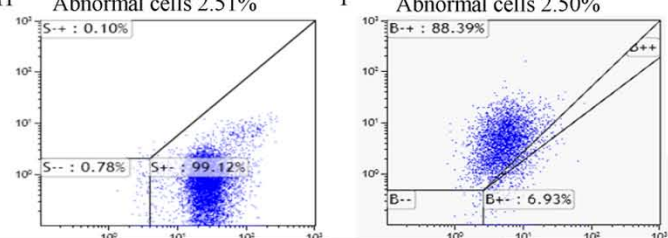

Kappa

Figure 3. Abnormal plasma cells detected using in MFC. (A) Plasma cells (0.03\%) with an abnormal phenotype were detected using MFC (corresponding to the patient in Fig. 2B) with monoclonal Lambda light chain. (B-I) Data corresponding to the patients shown in Fig. 2C-J, respectively. (B) The patient presented with $2.42 \%$ of myeloma cells detected with a monoclonal $\kappa$ light chain antibody. (C) The patient presented with $2.06 \%$ of myeloma cells detected with a $\lambda$ light chain antibody. (D) The patient presented with $0.09 \%$ of myeloma cells detected using a $\lambda$ light chain antibody. (E) The patient presented with $0.24 \%$ of myeloma cells detected using a $\lambda$ light chain antibody. (F) The patient presented with $0.06 \%$ of myeloma cells detected using a $\kappa$ light chain antibody. $(\mathrm{G})$ The patient presented with $0.60 \%$ of myeloma cells detected using a $\kappa$ light chain antibody. $(\mathrm{H})$ The patient presented with $2.51 \%$ of myeloma cells detected using a $\kappa$ light chain antibody. (I) The patient presented with $2.50 \%$ of myeloma cells detected using a $\lambda$ light chain antibody. MFC, multiparameter flow cytometry.

negatively correlated with miR-451a expression $\left(\mathrm{R}^{\mathrm{s}}=-0.97\right.$ $\mathrm{P}<0.0001$; Fig. 1D). The plasma cells from patients with MM demonstrated an upregulated expression of IL-6R compared with the control subjects via western blotting. Furthermore, the expression levels increased gradually from stage I to stage III (Fig. 1E). IL-6R levels were strongly and negatively correlated with miR-451a expression $\left(\mathrm{R}^{\mathrm{s}}=-0.95 ; \mathrm{P}<0.001\right.$; Fig. $\left.1 \mathrm{~F}\right)$.

After two courses of consolidation chemotherapy, 19 patients achieved complete remission (CR). In the 2-year follow-up period, MRD was observed using MFC and changes in circulating miR-451a expression were evaluated. For 10 patients, the expression of circulating miR-451a remained steady in the follow-up period (seven patients with R-ISS stage I, two with R-ISS stage II and one with R-ISS stage III). The change in miR-451a expression was $<50 \%$. The 10 patients were in continuous CR (Fig. 2A). However, the other nine patients (two patients with R-ISS stage I, three with R-ISS stage II and four with R-ISS stage III) demonstrated an abrupt decrease in circulating miR-451a expression during the follow-up period after CR (Fig. 2B-J). The value at the observation point was $>50 \%$ lower compared with the value measured 3 months prior $(50 \%$; Fig. 2G-J). The turning points in the trend appeared 4-8 weeks before abnormal plasma cells were obtained via MFC (Fig. 3A-I). Thus, when MFC results were still negative, significant changes in circulating miR-451a expression had occurred. Subsequently, clinical relapse occurred in these nine patients.

$I L-6 R$ is a direct target of miR-451a. Significantly higher miR-451a expression was observed in the mimic group compared with the mimic control cells and in non-transfected cells (both $\mathrm{P}<0.001$; Fig. 4A). The dual-luciferase reporter assay results revealed that co-transfection of the miR-451a mimic and IL-6R-wild-type (WT) resulted in decreased the luciferase activity compared with co-transfection of the mimic control and IL-6R-WT $(\mathrm{P}<0.001$; Fig. 4B). However, there was no effect of co-transfection of the miR-451a mimic and IL-6R-mut had no effect on the luciferase activity ( $\mathrm{P}>0.05$; Fig. 4B). Furthermore, the overexpression of miR-451a significantly downregulated IL-6R expression both at the mRNA $(\mathrm{P}<0.001$; Fig. 4C) and protein (Fig. 4D) levels. Collectively, these results suggested that IL-6R was a target gene of miR-451a and was negatively regulated by miR-451a (Fig. 4E).

\section{Discussion}

With the wide application of novel drugs, the depth and duration of remission in patients with MM are continuously improving, but patients still eventually relapse or progress (3). The heterogeneity of patients who relapse requires an individualized assessment of these patients to determine the timing and medication used for treatment. Due to the biological complexity and dynamic nature of therapy, sampling a single site of the disease is unable to fully assess the clonal complexity of multisite metastatic disease in patients (7). Therefore, cell-free nucleic acids in circulation are useful for detecting the genomic alterations present in patients with genetically heterogeneous diseases. In the current study, a circulating miRNA, miR-451a, was indicated to be an efficient approach to monitor dynamic tumor load and predict relapse.

MM has a unique characteristic, namely, myeloma cells are not evenly distributed in BM, which are called foci; this characteristic often causes false negative results for MRD (7). Furthermore, MM is characterized by a high level of heterogeneity, as subclones cause clonal evolution. Thus, one type of mutation does not effectively characterize most patients with MM (22). Although the R-ISS stratification system is a powerful prognostic tool to predict outcomes in patients with myeloma, it does not reflect the real time tumor load in real time (3). Therefore, a new marker that is universal, rapid and can be monitored in real time is urgently required. To conduct follow-up tests more conveniently and avoid false negatives, the present study attempted to develop a non-invasive approach. 
A

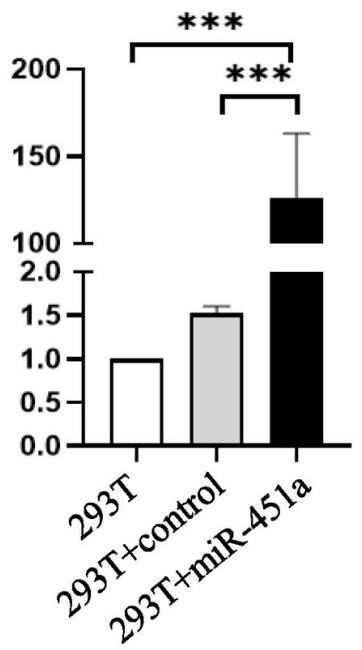

$\mathrm{B}$

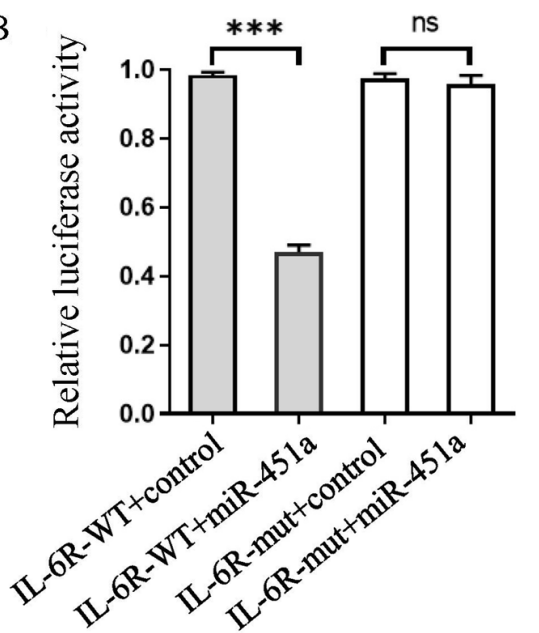

$\mathrm{C}$

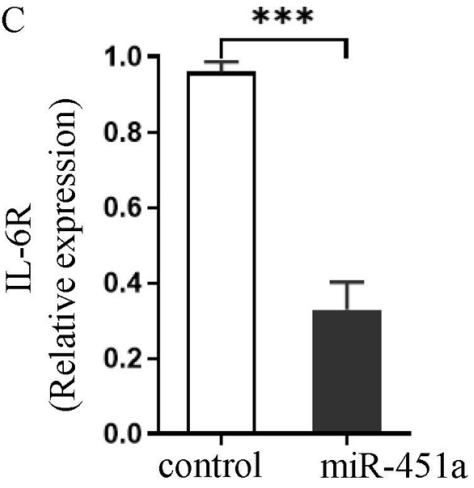

$\mathrm{D}$

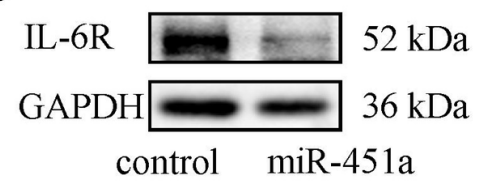

E

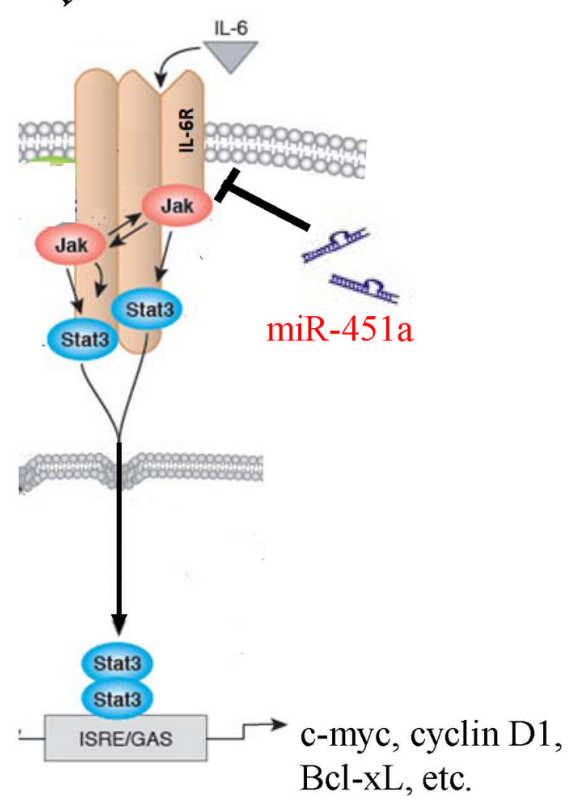

Figure 4. IL-6R is a direct target of miR-451a. (A) Expression of miR-451a in different groups. (B) Relationship between IL-6R and miR-451a was assessed via a dual-luciferase reporter assay. (C) Expression of the IL-6R mRNA in miR-451a mimic-transfected cells. (D) Western blotting demonstrating IL-6R levels in miR-451a mimic-transfected cells. (E) Associations between miR-451a, IL-6R and the JAK/STAT pathway. ${ }^{* * *} \mathrm{P}<0.001$; ${ }^{\text {ns }} \mathrm{P}>0.05$. ns, not significant; miR, microRNA; IL-6R, IL-6 receptor; WT, wild-type; mut, mutant.

A previous study reported that extracellular miRNA levels are stable in body fluids, and are retrievable and measurable from fresh or archived serum and plasma samples (23). In patients with cancer, the use of a less invasive method than tissue biopsy is needed, and it may lead to a major difference in clinical outcomes. Hence, a large number of studies have assessed the potential use of circulating miRNAs as biomarkers of various types of cancer, including breast cancer, gastric cancer, hepatocellular cancer and non-small cell lung cancer (24-26). Moreover, miRNA signatures in blood are similar in men and women, as well as in individuals of different ages (27). Circulating miRNAs originate from microvesicles (released by exocytosis), exosomes (formed by the invagination of the early endosome and released upon the fusion of the late endosome with the plasma membrane) and apoptotic vesicles and/or senescent bodies (27-29). In the current study, circulating miR-451a levels were measured using RT-qPCR, which is more sensitive compared with MFC, and more economical compared with next-generation sequencing. A stationary state of circulating miR-451a expression suggested remission, but an abrupt decrease in expression predicted relapse in the present study. This change often occurred prior to MFC and clinical relapse. However, the definition of an 'abrupt decrease' remains unclear. Based on the current results, 50\% may be the rate of change in miR-451a expression. However, the number of samples must be increased to identify the exact threshold.

The abnormal expression of miR-451a is observed in multiple solid malignant tumor types, and its expression is negatively correlated with the degree of malignancy (30-32). In the current research, the downregulation of miR-451a expression in the serum of patients with MM distinguished patients with MM from healthy individuals. Moreover, a decline in miR-451a expression is significantly correlated with poor clinical prognosis (33). This finding is consistent with the present study. However, the mechanism has not yet been elucidated. In humans and rats, miR-451a displays a tissue-specific expression pattern, and it is highly expressed in BM (34). As 
$\mathrm{BM}$ is part of the hematological system, miR-451a release into the blood may be more likely to occur. Long non-coding RNAs (lncRNAs) have been revealed to sponge miRNAs to decrease their expression (35). In terms of miR-451a, LINC00657 and AC084082.3 are shown to interact with miR-451a in BM (36). In MM, IL-6, a vital growth factor for myeloma cells, can induce the upregulation of several lncRNAs (37), and these lncRNAs may cause miR-451a dysregulation. However, which lncRNA can sponge miR-451a in MM remains unknown. In the present study, IL-6R was identified to be a target of miR-451a. Thus, it was indicated that miR-451a was associated with the IL-6R/JAK2/STAT3 pathway. However, whether the IncRNA that may sponge miR-451a is also associated with this pathway is yet to be elucidated, and will be examined in future studies.

The downstream mechanism of downregulated miR-451a that influences MM is not fully understood. In osteosarcoma, miR-451a inhibits the proliferation, migration and angiogenesis of osteosarcoma cells by silencing IL-6R (21). In the present study, miR-451a was negatively associated with the R-ISS stage, and negatively correlated with IL-6 and IL-6R. The target verification experiment identified the relationship between miR-451a and IL-6R. Therefore, miR-451a may function via the IL-6R/JAK2/STAT3 pathway. Previous studies have reported that miR-451a could change the levels of JAK2 and STAT3 phosphorylation to induce apoptosis in myeloma cells $(38,39)$. Therefore, this mechanism could apply to clinical transformation, and miR-451a has the potential to be a biomarker to predict the therapy outcome of a patient. In addition to the JAK2/STAT3 pathway, IL-6 could also promote the proliferation of myeloma cells via the Ras/MAPK pathway (40) and regulate the PI3K/AKT pathway to reduce apoptosis in MM cells (41). IL-6 interacts with VEGF to promote angiogenesis, migration and invasion (42). Moreover, numerous pathways activated by IL-6 could activate NF-Kb (43). Therefore, miR-451a may serve a role in other mechanisms, but these have yet to be confirmed.

In conclusion, miR-451a was associated with the R-ISS stage and tracked dynamic changes in the tumor. Circulating miR-451 could reflect the tumor load in real time and may be used to monitor MRD in patients with MM. An early warning sign, especially a non-invasive method, will allow for the anticipation of the disease. For instance, patients may start treatment if there is a dramatic and rapid decrease in miR-451a. However, if the rate of decline is slow, observation is recommended. Although the number of cases in the current study are limited, the available data demonstrate the value of miR-451a in early recurrence after chemotherapy in patients with MM. In the future, the sample size will continue to further enlarged to clarify and determine the prognostic value of miR-451a.

\section{Acknowledgements}

The manuscript was based on an abstract appearing in the 2019 Abstract Proceedings book of the ISLH (International Society of Laboratory Hematology; Volume 41, Issue S2; abstract no. 208). The authors appreciate the advice provided by Mr. Yi Shi (Sichuan Academy of Medical Sciences and Sichuan Provincial People's Hospital, School of Medicine, University of Electronic Science and Technology of China), who assisted with analyzing circulating miR-451a expression.

\section{Funding}

This work was supported by the Health Commission of Sichuan Province (grant no. 150193), the Commission of the Cardre Health Care in Sichuan Province (grant no. 2017-228), the Science and Technology Department of Sichuan Province (grant no. 19YJ0593), the Sichuan Provincial People's Hospital (grant no. 2018LY03), the Chengdu Science and Technology Bureau (grant no. 2015-HM01-00470-SF) and the Science and Technology Department of Sichuan Province (grant no. 2020YFS0433).

\section{Availability of data and materials}

The datasets used and/or analyzed during the current study are available from the corresponding author on reasonable request.

\section{Authors' contributions}

LZ, HJ, ZX, MZ, DC, YH, JZ, TJ and JC participated in the design, interpretation of the studies, analysis of the data and review of the manuscript. $\mathrm{LZ}, \mathrm{XJ}$ and $\mathrm{ZX}$ conducted the experiments. MZ conducted statistical analysis. DC and $\mathrm{YH}$ followed up the patients. LZ, JZ, TJ and JC wrote the manuscript. All authors read and approved the final manuscript.

\section{Ethics approval and consent to participate}

This study was performed with the approval of the Institutional Review Board of Sichuan Provincial People's Hospital of China. Informed written consent was collected from each eligible patient, and the whole study was performed in accordance with the Declaration of Helsinki.

\section{Patient consent for publication}

Not applicable.

\section{Competing interests}

The authors declare that they have no competing interests.

\section{References}

1. Palumbo A and Anderson K: Multiple myeloma. N Enq J Med 364: 1046-1060, 2011.

2. Rajkumar SV: Multiple myeloma: Every year a new standard? Hematol Oncol 37 (Suppl 1): S62-S65, 2019.

3. Kumar SK, Rajkumar V, Kyle RA, van Duin M, Sonneveld P, Mateos MV, Gay F and Anderson KC: Multiple myeloma. Nat Rev Dis Primers 3: 17046, 2017.

4. Tandon N, Rajkumar SV, LaPlant B, Pettinger A, Lacy MQ, Dispenzieri A, Buadi FK, Gertz MA, Hayman SR, Leung N, et al: Clinical utility of the revised international staging system in unselected patients with newly diagnosed and relapsed multiple myeloma. Blood Cancer J 7: e528, 2017.

5. Martin T and Huff CA: Multiple myeloma: Current advances and future directions. Clin Lymphoma Myeloma Leuk 19: 255-263, 2019.

6. Kumar S, Paiva B, Anderson KC, Durie B, Landgren O, Moreau P, Munshi N, Lonial S, Bladé J, Mateos MV, et al: International myeloma working group consensus criteria for response and minimal residual disease assessment in multiple myeloma. Lancet Oncol 17: e328-e346, 2016.

7. Paiva B, García-Sanz R and San Miguel JF: Multiple myeloma minimal residual disease. Cancer Treat Res 169: 103-122, 2016. 
8. Furukawa M, Ohkawara H, Ogawa K, Ikeda K, Ueda K, Shichishima-Nakamura A, Ito E, Imai JI, Yanagisawa Y, Honma R, et al: Autocrine and paracrine interactions between multiple myeloma cells and bone marrow stromal cells by growth arrest-specific gene 6 cross-talk with interleukin-6. J Bio Chem 292: 4280-4292, 2017.

9. Chong PSY, Zhou J, Lim JSL, Hee YT, Chooi JY, Chung TH Tan ZT, Zeng Q, Waller DD, Sebag M and Chng WJ: IL6 promotes a STAT3-PRL3 feedforward loop via SHP2 repression in multiple myeloma. Cancer Res 79: 4679-4688, 2019.

10. Jurczyszyn A, Czepiel J, Biesiada G, Gdula-Argasińska J, Cibor D, Owczarek D, Perucki W and Skotnicki AB: HGF, sIL-6R and TGF- $\beta 1$ play a significant role in the progression of multiple myeloma. J Cancer 5: 518-524, 2014

11. Altuvia Y, Landgraf P, Lithwick G, Elefant N, Pfeffer S, Aravin A, Brownstein MJ, Tuschl T and Margalit H: Clustering and conservation patterns of human microRNAs. Nucleic Acids Res 33: 2697-2706, 2005.

12. Wang R, Wang ZX, Yang JS, Pan X, De W and Chen LB MicroRNA-451 functions as a tumor suppressor in human non-small cell lung cancer by targeting ras-related protein 14 (RAB14). Oncogene 30: 2644-2658, 2011.

13. Huang JY, Zhang K, Chen DQ, Chen J, Feng B, Song H, Chen Y, Zhu Z,LuL,De W, et al: MicroRNA-451: Epithelial-mesenchymal transition inhibitor and prognostic biomarker of hepatocelluar carcinoma. Oncotarget 6: 18613-18630, 2015.

14. Kim Y, Powathil G, Kang H, Trucu D, Kim H, Lawler S and Chaplain M: Strategies of eradicating glioma cells: A multi-scale mathematical model with MiR-451-AMPK-mTOR control. PLoS One 10: e114370, 2015.

15. Yuan J, Lang J, Liu C, Zhou K, Chen L and Liu Y: The expression and function of miRNA-451 in osteosarcoma. Med Oncol 32: $324,2015$.

16. Gits CM, van Kuijk PF, Jonkers MB, Boersma AW, Smid M, van Ijcken WF, Coindre JM, Chibon F, Verhoef C, Mathijssen RH, et al: MicroRNA expression profiles distinguish liposarcoma subtypes and implicate miR-145 and miR-451 as tumor suppressors. Int J Cancer 135: 348-361, 2014

17. Liu X, Zhang A, Xiang J, Lv Y and Zhang X: miR-451 acts as a suppressor of angiogenesis in hepatocellular carcinoma by targeting the IL-6R-STAT3 pathway. Oncol Rep 36: 1385-1392, 2016.

18. Dimopoulos M, Terpos E, Comenzo RL, Tosi P, Beksac M, Sezer O, Siegel D, Lokhorst H, Kumar S, Rajkumar SV, et al: IMWG. International myeloma working group consensus statement and guidelines regarding the current role of imaging techniques in the diagnosis and monitoring of multiple myeloma. Leukemia 23: 1545-1556, 2009.

19. Livak KJ and Schmittgen TD: Analysis of relative gene expression data using real-time quantitative PCR and the 2(-Delta Delta C(T)) method. Methods 25: 402-408, 2001.

20. Flores-Montero J, Sanoja-Flores L, Paiva B, Puig N, García-Sánchez O, Böttcher S, van der Velden VHJ,Pérez-Morán JJ, Vidriales MB, García-Sanz R, et al: Next generation flow for highly sensitive and standardized detection of minimal residual disease in multiple myeloma. Leukemia 31: 2094-2103, 2017.

21. Liu SY, Deng SY, He YB and Ni GX: MiR-451 inhibits cell growth, migration and angiogenesis in human osteosarcoma via down-regulating IL 6R. Biochem Biophys Res Commun 482: 987-993, 2017.

22. Corre J, Cleynen A, Robiou du Pont S, Buisson L, Bolli N, Attal M, Munshi N and Avet-Loiseau H: Multiple myeloma clonal evolution in homogeneously treated patients. Leukemia 32 : 2636-2647, 2018

23. Schwarzenbach H, Nishida N, Calin GA and Pantel K: Clinical relevance of circulating cell-free microRNAs in cancer. Nat Rev Clin Oncol 11: 145-156, 2014.

24. Filipów S and Łaczmański Ł: Blood circulating miRNAs as cancer biomarkers for diagnosis and surgical treatment response. Front Genet 10: 169, 2019.

25. Oura K, Fujita K, Morishita A,Iwama H, Nakahara M, Tadokoro T, Sakamoto T, Nomura T, Yoneyama H, Mimura S, et al: Serum microRNA-125a-5p as a potential biomarker of HCV-associated hepatocellular carcinoma. Oncol Lett 18: 882-890, 2019.

26. Hagrass HA, Sharaf S, Pasha HF, Tantawy EA, Mohamed RH and Kassem R: Circulating microRNAs-a new horizon in molecular diagnosis of breast cancer. Genes Cancer 6: 281-287, 2015.
27. Cui M, Wang H, Yao X, Zhang D, Xie Y, Cui R and Zhang X: Circulating MicroRNAs in cancer: Potential and challenge. Front Genet 10: 626, 2019.

28. Mollashahi B, Aghamaleki FS and Movafagh A: The roles of miRNAs in medulloblastoma: A systematic review. J Cancer Prev 24: 79-90, 2019.

29. Schwarzenbach $\mathrm{H}$ and Gahan PB: MicroRNA shuttle from cell-to-cell by exosomes and its impact in cancer. Noncoding RNA 5: 28, 2019.

30. Zeng T, Peng L, Chao C, Fu B, Wang G, Wang Y and Zhu X: miR-451 inhibits invasion and proliferation of bladder cancer by regulating EMT. Int J Clin Exp Pathol 7: 7653-7662, 2014.

31. Liu F, Bu Z, Zhao F and Xiao D: Increased T-helper 17 cell differentiation mediated by exosome-mediated microRNA-451 redistribution in gastric cancer infiltrated T cells. Cancer Sci 109: $65-73,2018$

32. Wang W, Zhang L, Wang Y, Ding Y, Chen T, Wang Y, Wang H, Li Y, Duan K, Chen S, et al: Involvement of miR-451 in resistance to paclitaxel by regulating YWHAZ in breast cancer. Cell Death Dis 8: e3071, 2017.

33. Rastgoo N, Abdi J, Hou J and Chang H: Role of epigenetics-microRNA axis in drug resistance of multiple myeloma. J Hematol Oncol 10: 121, 2017.

34. Ludwig N, Leidinger P, Becker K, Backes C, Fehlmann T, Pallasch C, Rheinheimer S, Meder B, Stähler C, Meese E and Keller A: Distribution of miRNA expression across human tissues. Nucleic Acids Res 44: 3865-3877, 2016.

35. Panoutsopoulou K, Avgeris M and Scorilas A: miRNA and long non-coding RNA: Molecular function and clinical value in breast and ovarian cancers. Expert Rev Mol Diagn 18: 963-979, 2018.

36. Balakrishnan I, Yang X, Brown J, Ramakrishnan A, Torok-Storb B, Kabos P, Hesselberth JR and Pillai MM: Genome-wide analysis of miRNA-mRNA interactions in marrow stromal cells. Stem Cells 32: 662-673, 2014

37. Binder S, Zipfel I, Friedrich M, Riedel D, Ende S, Kämpf C, Wiedemann K, Buschmann T, Puppel SH, Reiche K, et al: Master and servant: LINC00152-a STAT3-induced long noncoding RNA regulates STAT3 in a positive feedback in human multiple myeloma. BMC Med Genomics 13: 22, 2020.

38. De Oliveira MB, Fook-Alves VL, Eugenio AIP, Fernando RC, Sanson LFG, de Carvalho MF, Braga WMT, Davies FE and Colleoni GWB: Anti-myeloma effects of ruxolitinib combined with bortezomib and lenalidomide: A rationale for JAK/STAT pathway inhibition in myeloma patients. Cancer Lett 403: 206-215, 2017.

39. Brown R, Yang S, Weatherburn C, Gibson J, Ho PJ, Suen H, Hart D and Joshua D: Phospho-flow detection of constitutive and cytokine-induced pSTAT3/5, pAKT and pERK expression highlights novel prognostic biomarkers for patients with multiple myeloma. Leukemia 29: 483-490, 2015.

40. Gocke CB, McMillan R, Wang Q, Begum A, Penchev VR, Ali SA, Borrello I, Huff CA and Matsui W: IQGAP1 Scaffold-MAP kinase interactions enhance multiple myeloma clonogenic growth and self-renewal. Mol Cancer Ther 15: 2733-2739, 2016.

41. Mimura N, Hideshima T, Shimomura T, Suzuki R, Ohguchi H, Rizq O, Kikuchi S, Yoshida Y, Cottini F, Jakubikova J, et al: Selective and potent Akt inhibition triggers anti-myeloma activities and enhances fatal endoplasmic reticulum stress induced by proteasome inhibition. Cancer Res 74: 4458-4469, 2014.

42. Berenstein R, Nogai A, Waechter M, Blau O, Kuehnel A Schmidt-Hieber M, Kunitz A, Pezzutto A, Dörken B and Blau IW: Multiple myeloma cells modify VEGF/IL-6 levels and osteogenic potential of bone marrow stromal cells via Notch/miR-223. Mol Carcinog 55: 1927-1939, 2016.

43. Matthews GM, de Matos Simoes R, Dhimolea E, Sheffer M, Gandolfi S, Dashevsky O, Sorrell JD and Mitsiades CS: NF- $\kappa$ B dysregulation in multiple myeloma. Semin Cancer Biol 39: 68-76, 2016.

This work is licensed under a Creative Commons Attribution-NonCommercial-NoDerivatives 4.0 International (CC BY-NC-ND 4.0) License. 